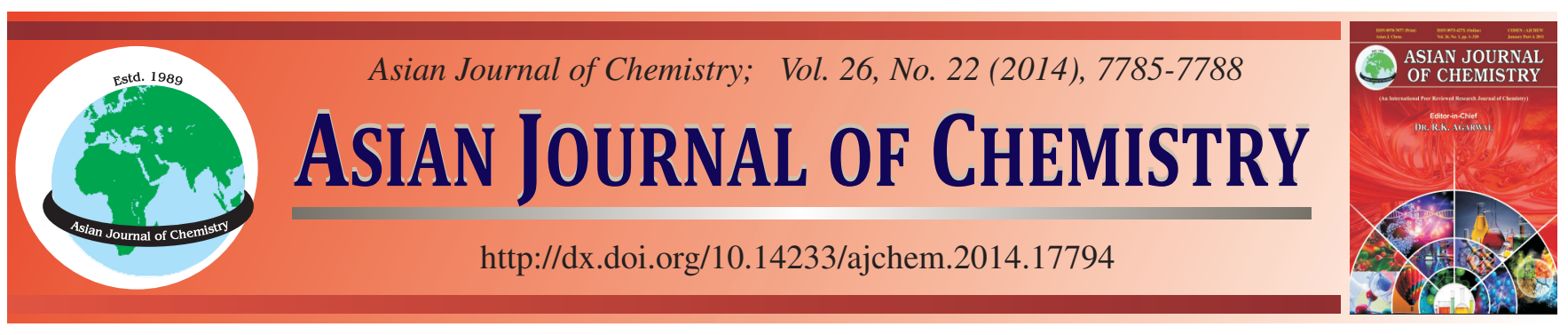

\title{
Theoretical Studies of Reaction Mechanism of 1,3-Indandione with (Z)- $N$-Methyl- $C$-Phenylnitrone by Metadynamic Approach
}

\author{
JAMAL LASRI $^{1,2, *}$ and GRZEGORZ GAJEWSKI ${ }^{3, \dagger}$
}

${ }^{1}$ Department of Chemistry, College of Science and Arts, King Abdulaziz University, P.O. Box 344 Rabigh 21911, Kingdom of Saudi Arabia ${ }^{2}$ Centro de Química Física Molecular, Instituto Superior Técnico, University of Lisbon, Av. Rovisco Pais, 1049-001, Lisbon, Portugal

${ }^{3}$ Research Center for Applied Sciences, Academia Sinica, 128 Academia Road, Section 2, Nankang 115-29, Taipei, Taiwan

*Corresponding author: Tel: +966 560247664; E-mail: jlasri@kau.edu.sa

DFT-based computational study of the reaction mechanism of the acyclic nitrone $(Z)$ - $N$-methyl- $C$-phenylnitrone (1) with 1,3 -indandione (2) by using the metadynamic method within the CPMD package was investigated. Two theoretical simulations have been performed to study this reaction. The first one corresponds to the formation of the alkylhydroxylamine intermediate, while the second one concerns the $\mathrm{C}-\mathrm{N}$ bond cleavage which leads to the elimination of $\mathrm{N}$-methylhydroxylamine and formation of 2-benzylidene-2H-indene-1,3-dione (2'). The gas phase calculations predicted that the reaction is slightly endothermic $(+2.71 \mathrm{kcal} / \mathrm{mol})$. The computed energy profile also indicated that the ketone-enol tautomerization in this reaction determines the height of the free energy barrier which is about $30 \mathrm{kcal} / \mathrm{mol}$.

Keywords: Metadynamic method, Acyclic nitrones, $\beta$-Diketones, Endiones.

\section{INTRODUCTION}

The synthesis of substituted olefins in a stereoselective fashion is still a challenging task in organic chemistry. The synthesis of olefins involves elimination reactions, which usually affords a mixture of $\mathrm{E}$ and $\mathrm{Z}$ isomers with the predominance of one isomer (Zaytzev or Hofmann rules), however, the high stereoselectivity is rare ${ }^{1}$. The first successful methods for the stereoselective synthesis of olefins include the Knoevenagel condensations ${ }^{2}$, the McMurry ${ }^{3}$ and Wittig ${ }^{4}$ reactions from carbonyl compounds (e.g. aldehydes or ketones), which were also proved to be accelerated by focused microwave irradiation (M.W. $)^{5}$. Olefin metathesis showed to be a powerful synthetic methodology within both functionalized and unfunctionalized olefins ${ }^{6}$.

The traditional Knoevenagel condensation is carried out by reacting methylene active compounds containing two electron-acceptor groups, such as $\beta$-ketoesters, malonates, cyanoacetates and malononitrile, with ketones or aldehydes in the presence of a base $\mathrm{e}^{2,7}$. The condensation reaction can also be assisted by Lewis acids such as $\mathrm{TiCl}_{4}{ }^{8}, \mathrm{ZnCl}_{2}{ }^{9}$, $\mathrm{CeCl}_{3} \cdot 7 \mathrm{H}_{2} \mathrm{O} / \mathrm{NaI}^{10}$, and $\mathrm{HClO}_{4}-\mathrm{SiO}_{2}{ }^{11}$. The Knoevenagel products are valuable intermediates for further reactions, such as Diels-Alder and Michael additions ${ }^{12}$.
The employment of $\beta$-diketones as starting materials for the Knoevenagel reaction is, in fact, little explored ${ }^{12,13}$, because these compounds are less reactive than those normally used in view of their ability to form a stable cyclic enol. Some research groups reported that magnesium perchlorate ${ }^{14}$ or L-proline ${ }^{15}$ shown to be efficient catalysts for the syntheses of trisubstituted olefins via a Knoevenagel condensation between $\beta$-diketones and aldehydes.

In one of our previous publications, we reported that the reaction of the acidic $\alpha-\mathrm{CH}_{2}$ organonitriles $\mathrm{NCCH}_{2} \mathrm{R}$ bearing an electron-acceptor substituent ( $\left.\mathrm{R}=\mathrm{CO}_{2} \mathrm{Me}, \mathrm{SO}_{2} \mathrm{Ph}, \mathrm{COPh}\right)$ with acyclic nitrones ( $(Z)-N$-methyl- $C$-arylnitrones) leads to the formation of E-cyanoolefins ${ }^{16}$. Furthermore, we recently reported a novel strategy for the syntheses of olefins by reaction of (Z)- $N$-methyl- $C$-arylnitrones with $\beta$-diketones without the use of any catalyst or promoter ${ }^{17}$.

Herein, the mechanism of the reaction of 1,3-indandione with (Z)- $N$-methyl- $C$-phenylnitrone is investigated and discussed by using the recently introduced metadynamic method ${ }^{18-20}$.

\section{COMPUTATIONAL DETAIL}

The ab initio molecular dynamics (AIMD) simulations have been performed using the Car-Parrinello scheme along with efficient Nose-Hoover chain thermostats ${ }^{21}$ for nuclei and 
electronic orbitals in a cubic box of $18 \AA$ subject to periodic boundary conditions. The simulations have been carried with the Vanderbilt ultra-soft pseudopotentials ${ }^{22}$ with a cutoff of 35 Ry and with the Perdew-Burke-Ernzerhof $(\mathrm{PBE})^{23}$ functional and a fictitious electron mass of $700 \mathrm{au} . \mathrm{T}=350 \mathrm{~K}$. Furthermore a time step of 5 a.u. ( $0.12 \mathrm{fs}$ ) has been used.

To reduce the numbers of collective variables $(\mathrm{CV})$ during the single metadynamic calculation two theoretical simulations have been performed. The first one corresponds to reaction $\mathrm{A}$ (Scheme-I) with formation of the alkylhydroxylamine intermediate. The second one concerns the reaction B (Scheme-I) where $\mathrm{C}-\mathrm{N}$ bond cleavage leads to the elimination of $\mathrm{N}$-methylhydroxylamine and formation of 2-benzylidene- $2 \mathrm{H}$-indene1,3-dione 2'. For the first metadynamic simulations two collective variables (CV) have been chosen. The first $\mathrm{CV}(\mathrm{A} 1)$ $=\mathrm{r}(\mathrm{C} 1-\mathrm{H} 1)-\mathrm{r}(\mathrm{C} 1-\mathrm{H} 2)$ is the difference between bond lengths of $\mathrm{C}-\mathrm{H}$ bonds in $\mathrm{CH}_{2}$ group and measures the progress of removal of one of the acidic methylene protons and the second $\mathrm{CV}(\mathrm{A} 2)=\mathrm{r}(\mathrm{C} 1-\mathrm{C} 2)$ is the distance between 1,3-indandione (2) and (Z)- $N$-methyl- $C$-phenylnitrone (1) (Fig. 1). Additionally, a repulsive potential wall has been placed at around $6 \AA$ at first and then at $5 \AA$ for the second collective variables in order to limit the distance between the two molecules.
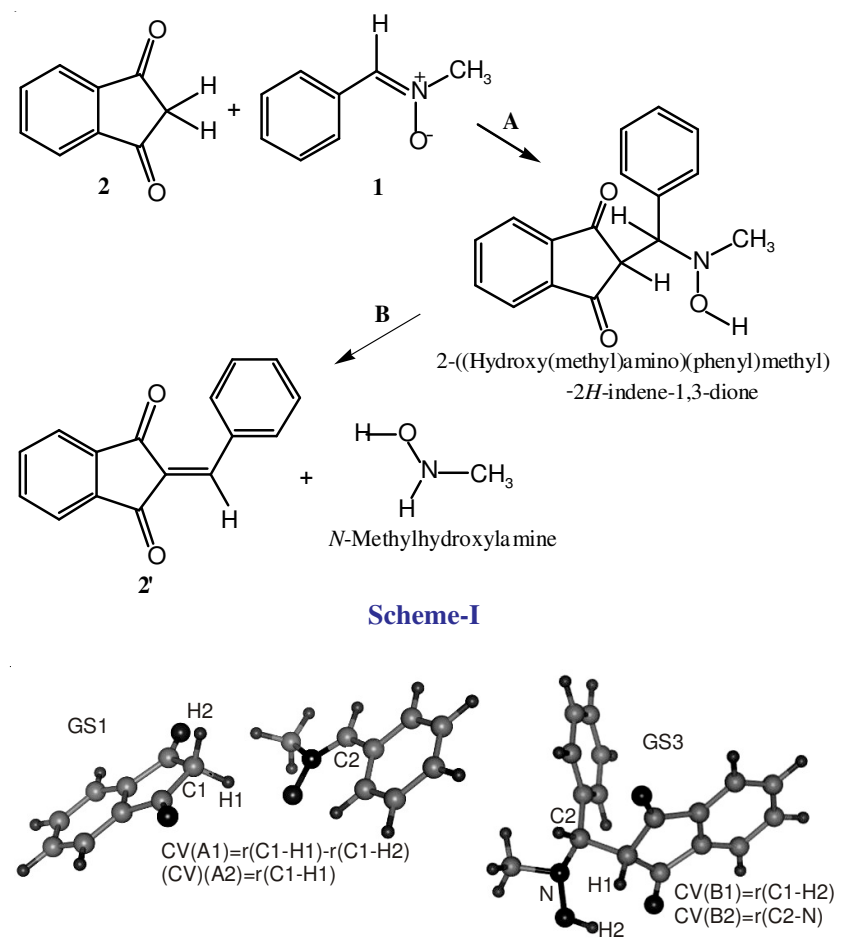

Fig. 1. Geometry of GS1 and GS3, and CVs used in the metadynamic simulations of reactions $\mathrm{A}$ and $\mathrm{B}$

The temperature of the system was slowly raised from zero to $350 \mathrm{~K}$ and then equilibrated for over $1 \mathrm{ps}$. In the next step, new inverted Gaussians have been added after at least $100 \mathrm{MD}$ steps provided that the displacement of the collective variable exceeded 0.1 . Otherwise, the addition has been forced after $200 \mathrm{MD}$ steps. For exploration of the reactant well in metadynamic spherical 2D Gaussian, potentials have been used with width $\mathrm{w}=0.22$ and height $\mathrm{h}=0.005$ a.u. After MD simulations A and B have reached transitions states TS1 (Scheme-II) and TS4 (Scheme-III), respectively, the width and height of added Gaussians have been reduced by the factor 2. Other parameters associated with the simulations have been set as follows: the fictitious mass of the collective variable was selected to be $20 \mathrm{amu}$, the corresponding coupling constant being 0.2 au.

Based on time evolution of collective variable's in these simulations, several structures have been chosen from metadynamic trajectories in proximity of ground and transition states. Further they have been used as starting points to optimization of nuclear geometries of ground states (GS) and transition states (TS). The limited-memory Broyden-FletcherGoldfarb-Shanno (L-BFGS) method has been used to search the ground states and with the microiterative scheme using the partitioned rational function optimizer (P-RFO) to search the transition states. The convergence criteria of $10^{-6}$ and $10^{-4}$ atomic unit have been used for gradient of the wavefunction and the ions, respectively. The mechanism of the reaction B (Scheme-I) has been simulated using also two collective variables. The distance $\mathrm{C} 1-\mathrm{H} 2$ has been chosen as the first collective variable to show the abstraction of $\mathrm{H} 2$ proton and the second collective variables $(\mathrm{CV}(\mathrm{B} 2)=\mathrm{r}(\mathrm{C} 2-\mathrm{N}))$ as indicator of the $\mathrm{C}-\mathrm{N}$ bond cleavage (Fig. 1).

\section{RESULTS AND DISCUSSION}

The recently introduced metadynamic method ${ }^{18-20}$ is a powerful tool to compute the multidimensional free energy surface of intrinsically concerted reactions. This method allows localizing the lowest free energy path that connects the stable reactant and product states. This path represents the most probable reaction mechanism, similar to the zero temperature intrinsic reaction coordinate, but also includes finite temperature effects. In this algorithm the normal evolution of the system is biased by a history-dependent potential constructed as a sum of Gaussians centred along the trajectory followed by a suitably chosen set of collective variables. That approach at DFT level incorporated in the $\mathrm{CPMD}^{24,25}$ package was used to find the lowest free energy reaction path, as well as structures of reactants, products and transitions states. It also allows establishing the enthalpy and activation barrier of these reactions. As opposed to more conventional sampling methods, the metadynamics technique allows us to map out the relevant multidimensional free-energy surface, which describes the concerted general-base mechanism in question.

The reaction of an acyclic nitrone ( $Z$ ) $-N$-methyl- $C$-phenylnitrone (1) with a 1,3-indandione (2), which constitutes a simple alternative method for the preparation of a pure endione 2-benzylidene-2H-indene-1,3-dione ( $\left.\mathbf{2}^{\prime}\right)$ without the use of any catalyst or promoter (Scheme-I), was investigated theoretically. Fig. 2 plots the time evolution of the two collective variables obtained from the first simulation. From this plot and analysis of the trajectories of substrates during the simulation, a full mechanism was found (Scheme-II). Steps I and II correspond to the ketone to enol conversion of 1,3-indandione (2). A proton in the $\alpha$-position relatively to a carbonyl group is more acidic than that in a typical $\mathrm{C}-\mathrm{H}$ bond due to the electron-withdrawing effect of the carbonyl and the tautomerization is catalyzed by a base, in this case the $\mathrm{O}$ atom of the nitrone. Experimental and theoretical results ${ }^{26}$ show that the 

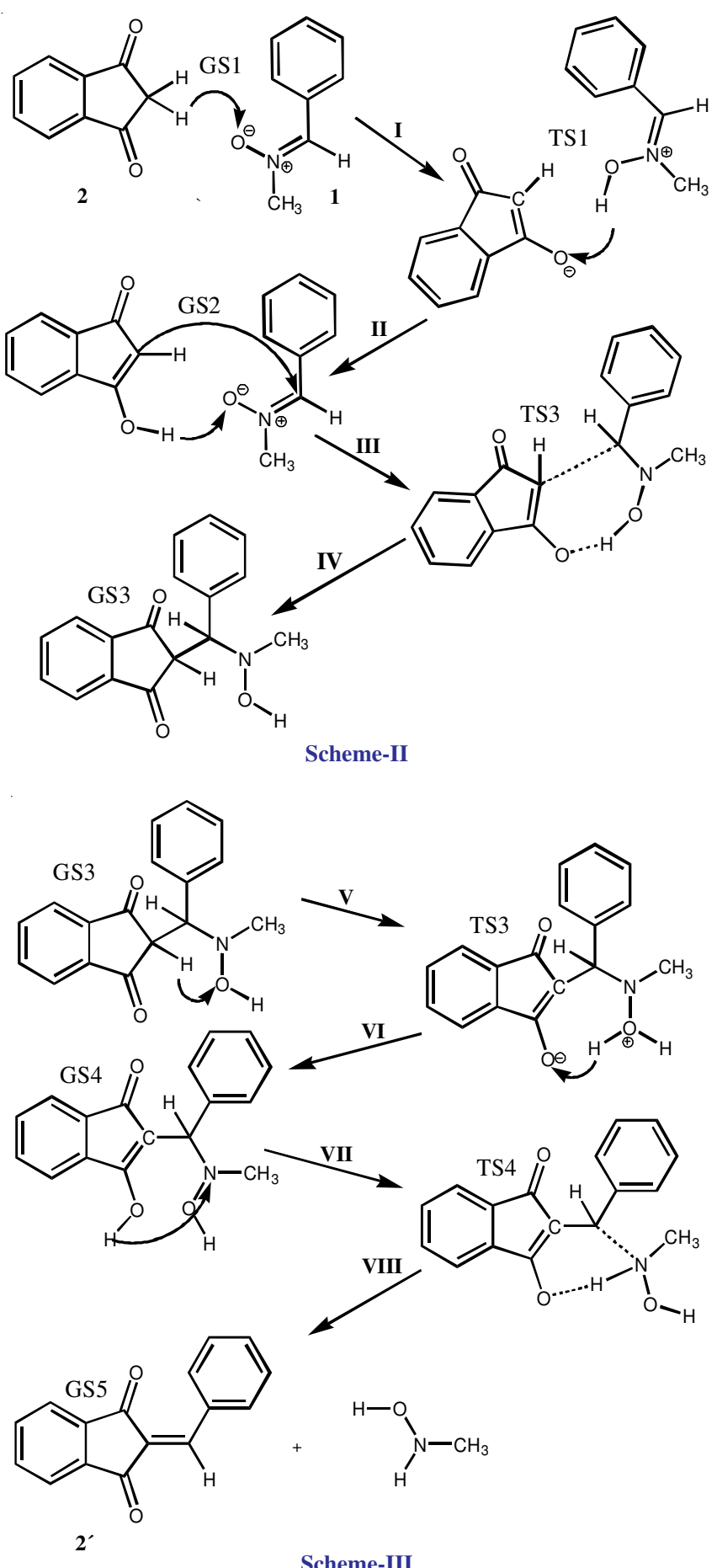

Scheme-III

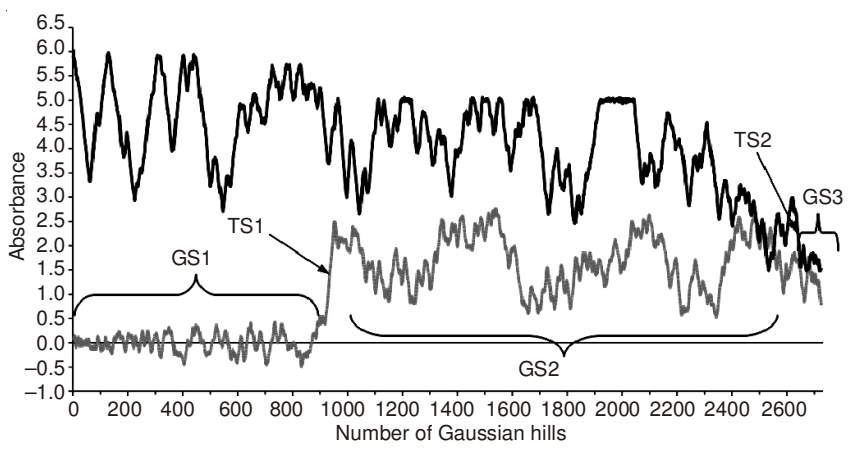

Fig. 2. Time evolution of collective variables during the simulation of reaction A. Red line: collective variable (A1); black line: collective variable (A2) negative potential is concentrated around this atom, while the rest of the molecule has a positive electrostatic potential. The system 1,3-indandione 2/( $Z$ )- $N$-methyl- $C$-phenylnitrone (1) (GS1) in the ketone form is $4.42 \mathrm{kcal} / \mathrm{mol}$ more stable than that with the enol form (GS2).

In steps III and IV the nucleophilic $\alpha$-carbon of the enol attacks the $\mathrm{C}=\mathrm{N}$ bond, which has an enhanced electrophilicity with respect to imines and other azomethines ${ }^{27}$, and forms a $\mathrm{C}-\mathrm{C}$ bond in the 2-[\{hydroxy(methyl)amino $\}$ (phenyl)methyl]$1 \mathrm{H}$-indene-1,3(2H)-dione intermediate (GS3). The calculated relative energy of this molecule is $3.42 \mathrm{kcal} / \mathrm{mol}$ above the energy of the 1,3-indandione (2)/(Z)- $N$-methyl- $C$-phenylnitrone (1) system (GS1).

The mechanism of the reaction B (Scheme-I) was simulated using also two collective variables. The time behaviour of the collective variables collective variable (B1) and collective variable (B2) (Fig. 3) and trajectories of all atoms during the metadynamics allow to describe the mechanism of reaction B (Scheme-III). In steps V and VI we can see again the ketoneenol tautomerization of 2-[\{hydroxy(methyl)amino $\}$ (phenyl)methyl]-1H-indene-1,3(2H)-dione (GS3) catalyzed by the $\mathrm{OH}$ group. The enol tautomer (GS4) is about $1.61 \mathrm{kcal} / \mathrm{mol}$ less stable than the ketone and $5.03 \mathrm{kcal} / \mathrm{mol}$ than 1,3-indandione (2)/(Z)- $N$-methyl- $C$-phenylnitrone (1) system (GS1).

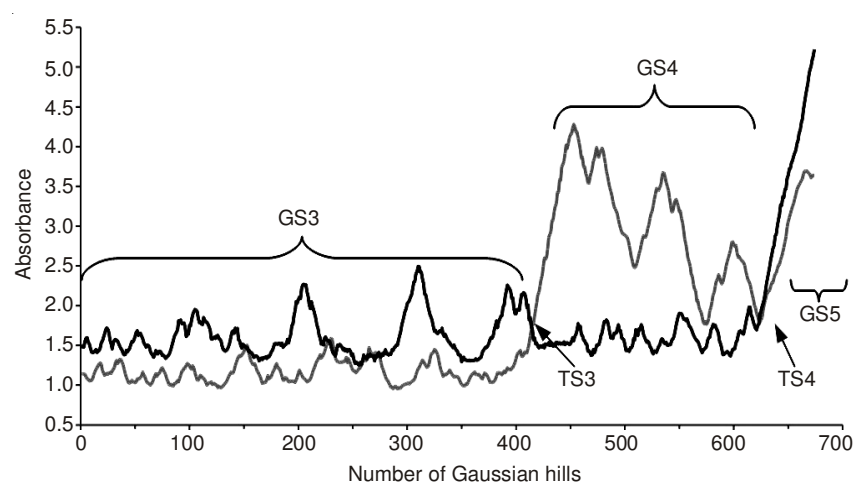

Fig. 3. Time evolution of collective variables during the simulation of reaction B. Black line: collective variable (B1); red line: collective variable (B2)

In the last two steps (VII and VIII), breaking of C-N and $\mathrm{O}-\mathrm{H}$ bonds with the concomitant creation of $\mathrm{C}=\mathrm{C}$ and $\mathrm{N}-\mathrm{H}$ bonds lead to the elimination of $N$-methylhydroxylamine and the formation of 2-benzylidene- $2 H$-indene-1,3-dione 2' (GS5). The calculated energy of the 2-benzylidene- $2 H$-indene- 1,3 dione $2^{\prime} / \mathrm{N}$-methylhydroxylamine system (GS5) is $2.71 \mathrm{kcal} /$ mol above the energy of the substrates (GS1).

Fig. 4 presents the equilibrium geometries of all ground and transition states in reactions $\mathrm{A}$ and $\mathrm{B}$. The relative energy levels of substrates, products and intermediates are shown in Fig. 5. The energy profile of this reaction predicts that it is slightly endothermic $(+2.71 \mathrm{kcal} / \mathrm{mol})$ and disfavoured at the gas phase but the theoretical approach used in our calculations is not including solvent effects as well as specific conditions (high pressure) for this reaction.

The energy profile indicates that the activation energy amounts to almost $30 \mathrm{kcal} / \mathrm{mol}$ and is associated to the ketoneenol tautomerization of 1,3-indandione 2 (Step I and II). Only a slightly lower barrier $(25 \mathrm{kcal} / \mathrm{mol})$ is associated to the 


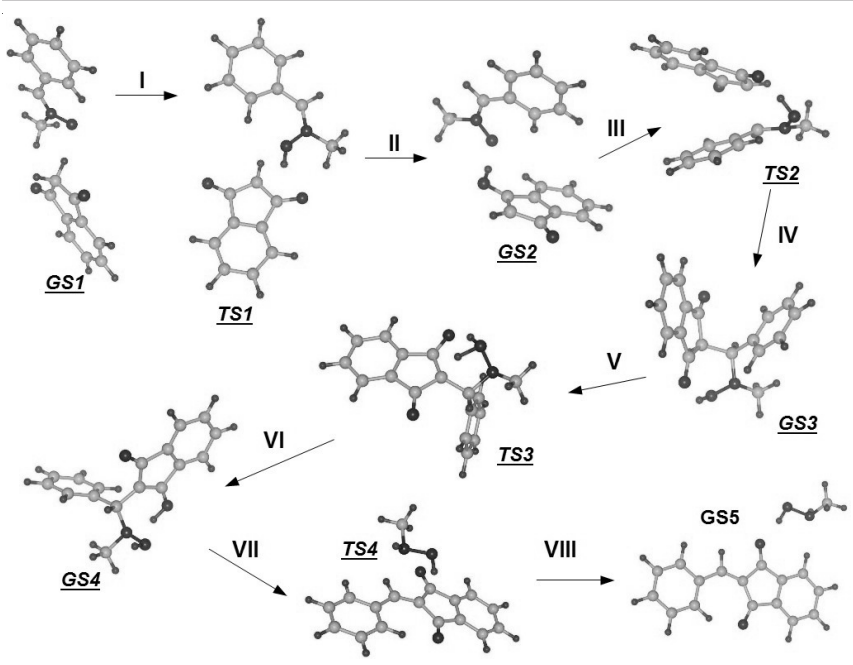

Fig. 4. Equilibrium geometries of ground and transition states involved in the reaction mechanism (Schemes-II and III)

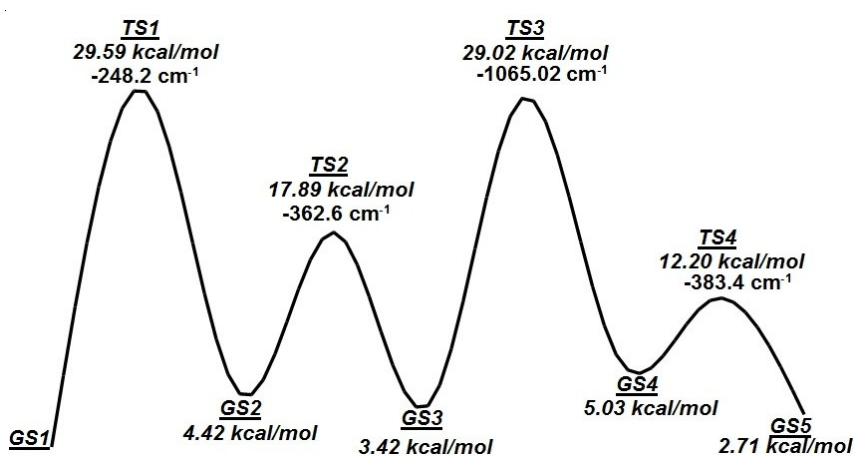

Fig. 5. Reaction free energy profile and imaginary frequencies of transition states

ketone-enol tautomerization of 2-[\{hydroxy(methyl)amino $\}$ (phenyl)methyl]-1H-indene-1,3(2H)-dione (steps V and VI).

\section{Conclusion}

In conclusion, we studied the mechanism of the reaction of acyclic nitrone (Z)- $N$-methyl- $C$-phenylnitrone (1) with 1,3indandione (2) by using the metadynamic method DFT-based computational study. This method was used to find the lowest free energy reaction path and to predict the structures of reactant, products and transition states as well as the enthalpy and activation barriers of this reaction. The first step proceeds with the formation of enol form of (2) which has higher energy than the corresponding keto form by $4.42 \mathrm{kcal} / \mathrm{mol}$. This step is followed by the formation of the $\mathrm{C}-\mathrm{C}$ bond via the nucleophilic attack of the $\alpha$-carbon of the enol form on the $\mathrm{C}=\mathrm{N}$ bond of (1) leading to the formation of the 2-[\{hydroxy(methyl)amino $\}$ (phenyl)methyl]-2H-indene-1,3-dione which is higher in energy than the initial reactants (1) and (2) by $3.42 \mathrm{kcal} / \mathrm{mol}$. The second step is also started with ketone-enol tautomerization followed by breaking of $\mathrm{C}-\mathrm{N}$ and $\mathrm{O}-\mathrm{H}$ bonds and creation of $\mathrm{C}=\mathrm{C}$ and $\mathrm{N}-\mathrm{H}$ bonds to afford 2-benzylidene- $2 \mathrm{H}$ indene-1,3-dione $\left(\mathbf{2}^{\prime}\right)$ and $N$-methylhydroxylamine, respectively. The final product $\left(2^{\prime}\right)$ has higher energy than the substrates (1) and (2) by $2.71 \mathrm{kcal} / \mathrm{mol}$ indicating that the reaction is slightly endothermic in the gas phase. The ketone-enol tautomerization in this reaction determines the height of the free energy barrier (about $30 \mathrm{kcal} / \mathrm{mol}$ ).

\section{REFERENCES}

1. S. Nakatsu, T. Gubaidullin, V.A. Mamedov and S. Tsuboi, Tetrahedron, 60, 2337 (2004) (and references cited therein).

2. E. Knoevenagel, Berichte, 31, 2596 (1898).

3. J.E. McMurry, Chem. Rev., 89, 1513 (1989).

4. G. Wittig and U. Schöllkopf, Chem. Ber., 87, 1318 (1954).

5. A. Spinella, T. Fortunati and A. Soriente, Synlett, 93 (1997).

6. R.H. Grubbs, Handbook of Metathesis, Wiley-VCH: Weinheim, Germany (2003).

7. G. Jones, Org. React., 15, 204 (1967)

8. W. Lehnert, Tetrahedron, 30, 301 (1974).

9. P.S. Rao and R.V. Venkataratnam, Tetrahedron Lett., 32, 5821 (1991).

10. G. Bartoli, R. Beleggia, S. Giuli, A. Giuliani, E. Marcantoni, M. Massaccesi and M. Paoletti, Tetrahedron Lett., 47, 6501 (2006).

11. S. Kantevari, R. Bantu and L. Nagarapu, J. Mol. Catal. Chem., 269, 53 (2007).

12. D.B. Ramachary and C.F. Barbas III, Chem. Eur. J., 10, 5323 (2004).

13. R. Maggi, F. Bigi, S. Carloni, A. Mazzacani and G. Sartori, Green Chem., 3, 173 (2001).

14. G. Bartoli, M. Bosco, A. Carlone, R. Dalpozzo, P. Galzerano, P. Melchiorre and L. Sambri, Tetrahedron Lett., 49, 2555 (2008).

15. P. Goswami and B. Das, Tetrahedron Lett., 50, 897 (2009).

16. J. Lasri, M.A.J. Charmier, M. Haukka and A.J.L. Pombeiro, J. Org. Chem., 72, 750 (2007).

17. J. Lasri, G. Gajewski, M.F.C. Guedes da Silva, M.L. Kuznetsov, R.R. Fernandes and A.J.L. Pombeiro, Tetrahedron, 68, 7019 (2012).

18. B. Ensing, A. Laio, M. Parrinello and M.L. Klein, J. Phys. Chem. B, 109, 6676 (2005).

19. A. Laio and M. Parrinello, Proc. Natl. Acad. Sci. USA, 99, 12562 (2002).

20. A. Laio, A. Rodriguez-Fortea, F.L. Gervasio, M. Ceccarelli and M. Parrinello, Phys. Chem. B, 109, 6714 (2005).

21. S. Nose and M. Klein, Mol. Phys., 50, 1055 (1983).

22. D. Vanderbilt, Phys. Rev. B, 41, 7892 (1990).

23. J. Perdew, K. Burke and M. Ernzerhof, Phys. Rev. Lett., 77, 3865 (1996).

24. R. Car and M. Parrinello, Phys. Rev. Lett., 55, 2471 (1985).

25. CPMD, http://www.cpmd.org/, Copyright IBM Corp 1990-2008; Copyright Max-Planck-Institut für Festkörperforschung, Stuttgart (1997-2001).

26. D.E. Hibbs, J.R. Hanrahan, M.B. Hursthouse, D.W. Knight, J. Overgaard, P. Turner, R.O. Piltz and M.P. Waller, Org. Biomol. Chem., 1, 1034 (2003).

27. M. Lombardo and C. Trombini, Synthesis, 759 (2000). 